\title{
Erratum to: Re-irradiation of recurrent anaplastic ependymoma using radiosurgery or fractionated stereotactic radiotherapy
}

\author{
Taro Murai $^{1,2} \cdot$ Kengo Sato $^{3,4} \cdot$ Michio Iwabuchi $^{3} \cdot$ Yoshihiko Manabe $^{1} \cdot$ \\ Hiroyuki Ogino ${ }^{5}$ Hiromitsu Iwata $^{5} \cdot$ Koshi Tatewaki $^{4} \cdot$ Naoki Yokota $^{6} \cdot$ Seiji Ohta $^{5}$. \\ Yuta Shibamoto ${ }^{1}$
}

Published online: 28 January 2016

(C) Japan Radiological Society 2016

\section{Erratum to: Jpn J Radiol \\ DOI 10.1007/s11604-015-0511-5}

There were errors in a figure and a table in the article cited above.

Fig. 1: The letters "OS" were missing on the chart. The corrected Fig. 1 is shown here.

Table 4: In the column headed "OS (\%)", the present study should be "38", not " 53 ".

The authors regret the errors and any inconvenience they may have caused.

The online version of the original article can be found under doi:10.1007/s11604-015-0511-5.

Taro Murai

taro8864@yahoo.co.jp

Kengo Sato

ksato@iyars.com

Michio Iwabuchi

m.iwabuchi0628@gmail.com

Yoshihiko Manabe

nabe.ncu@gmail.com

Hiroyuki Ogino

oginogio@gmail.com

Hiromitsu Iwata

h-iwa-ncu@nifty.com

Koshi Tatewaki

ktatewaki1967@syck.jp

Naoki Yokota

n.yokota@ suzukake.or.jp

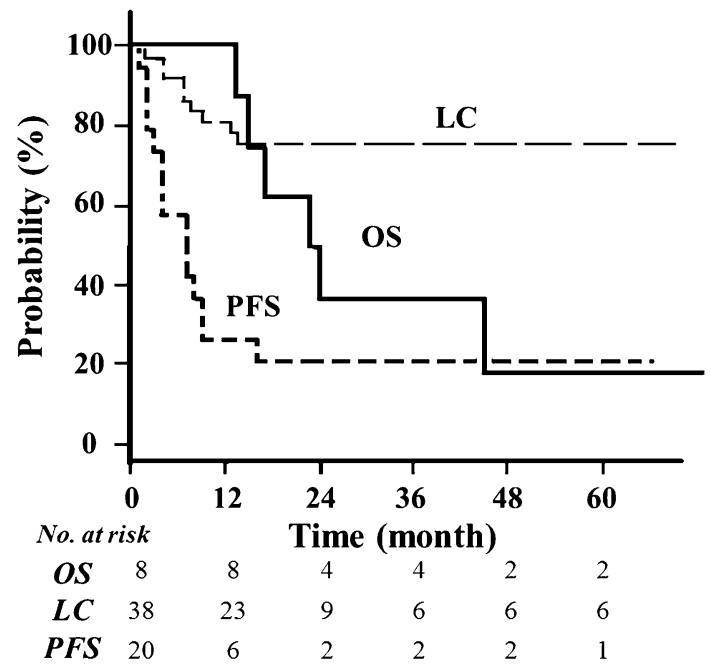

Fig. 1 OS, PFS, and LC curves. OS overall survival, $L C$ local control, $P F S$ progression-free survival

Seiji Ohta

seijiota@syck.jp

Yuta Shibamoto

yshiba@med.nagoya-cu.ac.jp

1 Department of Radiology, Nagoya City University Graduate School of Medical Sciences, 1 Kawasumi, Mizuho-cho, Mizuho-ku, Nagoya 467-8601, Japan

2 Department of Radiology, Tsushima City Hospital, Tsushima, Japan

3 Department of Neurosurgery, Japanese Red Cross Medical Center, Tokyo, Japan

4 Yokohama CyberKnife Center, Yokohama, Japan

5 Department of Radiation Oncology, Nagoya Proton Therapy Center, Nagoya City West Medical Center, Nagoya, Japan

6 Radiation Oncology Center, Suzukake Central Hospital, Hamamatsu, Shizuoka, Japan 